\title{
Assessment of Digital Image Correlation as a method of obtaining deformations of a structure under fluid load
}

\author{
J. Banks*, L. Marimon Giovannetti ${ }^{*}$, X. Soubeyran*, A.M. Wright**, S.R. Turnock*, \\ S.W. Boyd* \\ * Fluid Structure Interactions Group, Faculty of Engineering and the Environment, \\ University of Southampton, Southampton, SO16 7QF, UK \\ ** Wolfson Unit for Marine Technology and Industrial Aerodynamics, University of \\ Southampton, Southampton, SO17 1BJ, UK \\ E-mail: J.Banks@ soton.ac.uk
}

\begin{abstract}
Digital Image Correlation (DIC) is employed for the measurement of full-field deformation during fluid-structure interaction experiments in a wind tunnel. The methodology developed for the wind tunnel environment is quantitatively assessed. The static deformation error of the system is shown to be less than $0.8 \%$ when applied to a curved aerofoil specimen moved through known displacements using a micrometer. Enclosed camera fairings were shown to be required to minimise error due to wind induced camera vibration under aerodynamic loading. The methodology was demonstrated using a high performance curved foil, from a NACRA F20 sailing catamaran, tested within the University of Southampton RJ Mitchell, 3.5m x 2.4m, wind tunnel. The aerodynamic forces induced in the wind tunnel are relatively small, compared with typical hydrodynamic loading, resulting in small deformations. The coupled deflection and blade twist is evaluated over the tip region (80-100\% Span, measured from the root) for a range of wind speeds and angles of attack. Steady deformations at low angles of attack were shown to be well captured however unsteady deformations at higher angles of attack were observed as an increase in variability due to hardware limitations in the current DIC system. It is concluded that higher DIC sample rates are required to assess unsteady deformations in the future. The full field deformation data reveals limited blade twist for low angles of attack, below the stall angle. For larger angles, however, there is a tendency to reduce the effective angle of attack at the tip of the structure, combined with an unsteady structural response. This capability highlights the benefits of the presented methodology over fixed-point measurements as the three dimensional foil deflections can be assessed over a large tip region. In addition, the methodology demonstrates that very small deformations and twist angles can be resolved.
\end{abstract}

Keywords: Wind tunnel tests; Digital Image Correlation; Fluid Structures Interaction; Composite materials; Aeroelastic tailoring 


\section{Nomenclature}

$\begin{array}{ll}\alpha & \text { foil angle of attack } \\ \theta & \text { camera stereo angle } \\ \varphi & \text { camera to tunnel transformation angle } \\ \rho & \text { air density } \\ s & \text { specimen distance to the cameras } \\ c_{b} & \text { blur circle } \\ c & \text { foil chord } \\ C_{D}, C_{Y}, C_{Z} & \text { drag, side and vertical force coefficients } \\ D_{F} & \text { distance from the lens to the far limit of the depth of field } \\ D_{N} & \text { distance from the lens to the near limit } \\ f & \text { the lens focal length } \\ L & \text { foil span } \\ N & \text { the lens } f \text {-number } \\ R e & \text { Reynolds Number } \\ V_{S} & \text { wind speed } \\ \text { Coordinate systems: } \\ x_{c}, y_{c}, z_{c} & \text { camera (DIC system) } \\ x_{t}, y_{t}, z_{t} & \text { tunnel } \\ x_{d}, y_{d}, z_{d} & \text { dynamometer (foil forces) }\end{array}$

\section{Introduction}

Fluid Structure Interaction (FSI) studies have been mainly focused on the coupled numerical modelling of structural deformation under fluid loading utilising Finite Element Analysis (FEA) and Computational Fluid Dynamics (CFD) for the structures and fluids respectively (Lee, Jhan and Chung 2012, Nicholls-Lee, Boyd and Turnock 2009, Fedorov, et al. 2009, Maheri, Noroozi and Vinney 2006). In isolation there are many methods for the validation of FEA and CFD. However, there is a lack of experimental validation data for FSI investigations associated with accurate measurement of structural deformation under fluid loading.

Wind tunnel testing provides a controlled environment for the fluid loading of structures. The forces developed during testing are acquired via a dynamometer. However, standard wind tunnel testing often employs stiff structures such that the geometry remains effectively constant with respect to fluid loading. In reality structures will deform with increasing fluid loading, particularly where fibre reinforced composites are employed. The current research focuses on the assessment of an experimental technique that can be used to quantify the deformation and the bend-twist coupling for aero-elastic tailored composite structures under fluid loading, i.e. FSI.

In the past two decades, the trend of investigating the potential applications of composites structures has widely increased. Composite materials not only present a high stiffness to weight ratio, but also a better fatigue resistance compared to metallic components (Lee and Lin 2004). Using the anisotropy of the material, it is possible to 
design components presenting elastic couplings that will enhance the performance of the whole structure (Veers and Bir 1998, V. Fedorov 2012, Shirk and Hertz 1986, Liu and Young 2009, Young 2008). Careful directional placement of fibres and design of the composite architecture can result in an additional coupled response that will affect the effective angle of attack of the foil structures. In order to investigate this process an accurate full-field technique is required to measure the deformation whilst under fluid loading.

The experimental method used is the highly established Digital Image Correlation (DIC) technique. DIC has been used at a variety of scales from high magnification (Crammond, Boyd and Dulieu-Barton 2013) to large-scale structures (McCormick and Lord 2012). This technique involves the use of digital cameras that register a series of images of a surface on which a randomised speckle pattern is applied. The key advantages are the use of simple equipment (i.e. cameras, lenses, lights and a computer), the fact that it is a non-contact measurement and its high fidelity of precision, (Zhengzong, et al. 2011). Within DIC software, the speckle pattern is mapped to calculate the deformed shape, thereby allowing the derivation of the deflections and strains of the investigated object, (Rastogi and Hack 2012). The use of a single camera allows for the measurement of deformation in a single plane normal to the camera, i.e. 2D DIC. The use of two cameras, in a stereo configuration, allows for the measurement of deformations both in the plane normal to the camera and out of plane, i.e. 3D DIC. For 3D DIC the angle between the cameras controls the measurement accuracy of the out of plane deformation (Sutton, Orteu and Schreier 2009, Zhengzong, et al. 2011, Rastogi and Hack 2012, Reu 2013). For a foil structure the difference in leading and trailing edge deformation, normal to the chord, determines the impact of the deflection on effective angle of attack. Therefore optimizing the stereo angle between the two cameras for out of plane accuracy is essential (Reu 2013, Reu 2012, Ke, et al. 2011). DIC has been widely used and validated for twodimensional and three-dimensional analysis of small specimens, (Zhengzong, et al. 2011, Rastogi and Hack 2012, Helm, McNeill and Sutton 1996). These are mostly analysed using small stereo angles ( $\theta$ up to $17^{\circ}$ ) as this increases the in-plane resolution (Reu 2013, $\mathrm{Ke}$, et al. 2011). Three-dimensional DIC for a wide range of stereo angles and lenses are presented by Ke et al. (2011) and Phillip Reu (2013) and deflections and strains are assessed with experimental values, showing the possibility of increasing the stereo angle up to $\theta=60^{\circ}$ for large out of plane deformations.

The current research focuses on the assessment of the DIC technique to measure the deformation of foil structures under fluid loading. All of the available parameters (lenses, stereo angle, stand-off distance and speckle pattern) are herein assessed to provide a DIC procedure suitable for a wind tunnel environment. The tested specimen (a curved daggerboard from the NACRA F20 Carbon catamaran) allows the DIC methodology to be assessed in challenging conditions due to its complex geometry and the fact that only small deformations are expected under aerodynamic loading. The presented methodology will be of use not only to high performance foils for catamarans, but also to wind turbine blades (Lin and Lai 2010, Fedorov, et al. 2009, Karaolis, Musgrove and Jenimidis 1988, Nicholls-Lee, Boyd and Turnock 2009), helicopter rotors (Ganguli and Chopre November-December, 1995, Murugan, Ganguli and Harursampath January-February, 
2008), propeller blades (Khan, et al. 2000, Lee and Lin 2004), ship rudders (Turnock and Wright 2000, Molland and Turnock 2007) and high performance racing cars (Thuwis, et al. 2010). At the time of writing, little research has been developed involving Digital Image Correlation (DIC) within a wind tunnel to evaluate a coupled FSI problem. Most of the research in this field is investigating the elastic deformations and corresponding aerodynamic response of micro air vehicles, (Albertani, et al. 2007, Carpernter, Ray and Albertani 2012, LaCroix, Gardiner and Ifju 2013). The present research aims to develop a methodology that accurately captures the passive deformation of a foil structure under a fluid loading condition. The developed approach will then be assessed over a range of foil conditions including both wind speed and angle of attack to determine its suitability for obtaining validation data for FSI cases in the future.

\section{Methodology}

\subsection{Wind Tunnel Setup}

The experiments were conducted in the $3.5 \mathrm{~m}$ x $2.4 \mathrm{~m}$ RJ Mitchell wind tunnel at the University of Southampton. Two sets of experiments were performed in order to assess the repeatability of the results and the robustness of the approach. The closed circuit tunnel operates at wind speeds of 4 to $40 \mathrm{~ms}^{-1}$ with less than $0.2 \%$ turbulence. A six component Nutem load cell balance is mounted on a turntable in the tunnel roof. Figure 1 shows the cross-section of the wind tunnel and the position of the dynamometer.

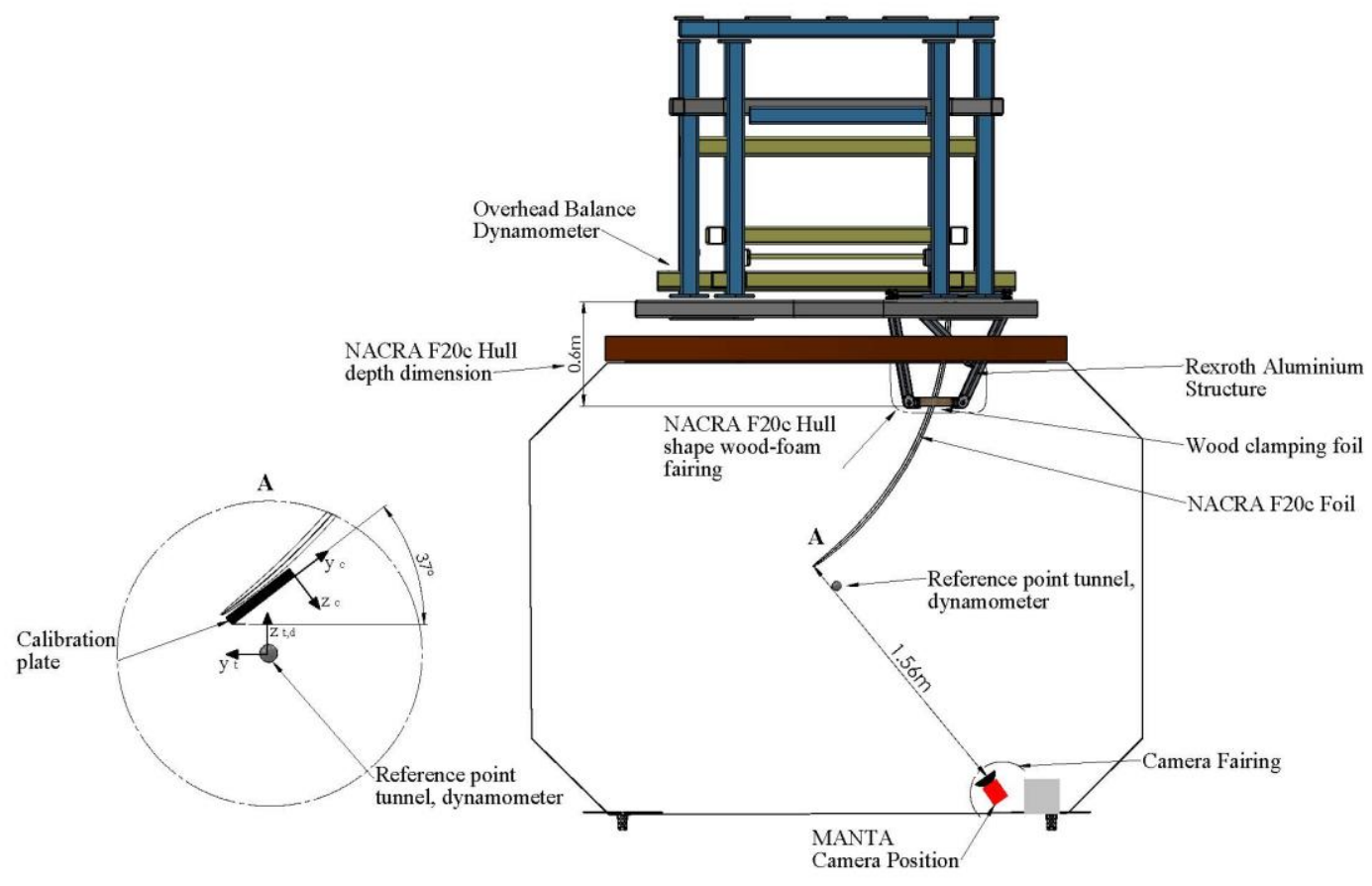

Figure 1: Wind tunnel arrangement showing specimen mounting system onto the dynamometer. Insert A illustrates the different coordinate systems relating to: the DIC camera system $x c, y c, z c$ (defined by the calibration plate), the tunnel $x t, y t$, zt (fixed) and the dynamometer $x d, y d$, zd (free to rotate about the vertical axis zt,d). 
The tested foil (NACRA F20 carbon daggerboard) has an exposed length of $1.1 \mathrm{~m}$ from root to tip in a straight line, a chord of $0.252 \mathrm{~m}$ and a thickness of $0.027 \mathrm{~m}$ before tapering at the tip. In order to attach the tested structure to the overhead balance it was necessary to build a rigid structure to hold the foil. The mounting frame was made of aluminium Rexroth@ bars supporting faired wooden blocks to clamp the foil at its root and a point $0.6 \mathrm{~m}$ below, as shown in Figure 1. The clamping arrangement of the foil replicates the boundary conditions at the bottom and top of the F20 hull, through which it passes.

The dynamometer and foil can be rotated about the vertical axis of the tunnel to allow an angle of attack to be applied. The dynamometer is designed to have a virtual centre (or reference point) 1.27 m below the tunnel roof, about which the moments and forces are measured. The tunnel and dynamometer coordinate system are the same for zero angle of attack, as depicted in Figure 1.

The mounting frame was attached to the overhead balance and enclosed by a representative hull-shaped fairing attached to the wind tunnel ceiling. This ensured that only the forces acting on the foil were recorded by the dynamometer, whilst providing similar flow conditions to a foil protruding from a catamaran hull, see Figure 2. The mounting frame was offset from the tunnel centreline so that the structure would rotate about a point near the blade tip. This ensured that as the angle of attack increased the blade tip would remain in the field of view of the DIC camera system. Figure 1, shows the wind tunnel and camera positions. It was possible to assess that the surface of the foil was at an angle of $37^{\circ}$ with respect to the tunnel floor in the wind tunnel axis.

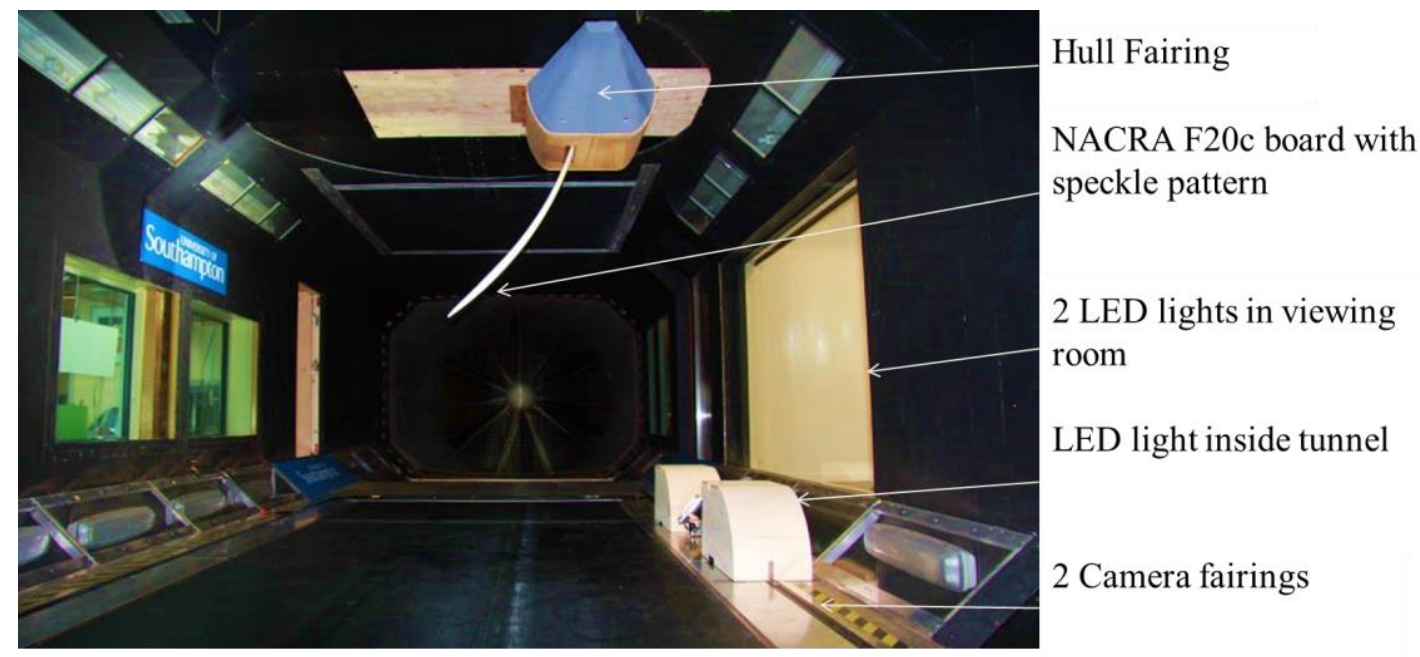

Figure 2: R.J. Mitchell wind tunnel working section, showing fairings and DIC equipment positions.

For each test condition of wind speed and foil angle of attack, the force and moment data was acquired at a frequency of $1 \mathrm{kHz}$ over a period of 10 seconds. Before each test condition all channels were zeroed to account for any systematic drift due to changing environmental conditions. The calibration of the dynamometer was checked by applying a known static load approximately normal to the foil surface. 


\subsection{Digital Image Correlation set up}

The speckle pattern used in DIC is commonly applied manually through the use of aerosol distributed paint. This produces a truly random speckle pattern but the quality is based on the skill of the user, the method of paint application and the distribution of speckle density and size (Crammond, Boyd and Dulieu-Barton 2013). Due to the scale of the testing in the present research, the relatively large stand-off distance of the camera to the structure and the relatively large field of view, a random digitally created speckle pattern was generated using the program ImageJ, allowing the size and density of the speckles to be controlled. A previous study by Soubeyran (2013) investigated the effect of different speckle patterns on displacement error for a DIC set-up similar to that used in the wind tunnel. A range of speckle sizes and densities were compared by providing a known out-of-plane displacement and assessing the error in the DIC displacement. A high-density pattern with a speckle size of approximately 6 pixels was found to minimize the error in displacements below $5 \mathrm{~mm}$, whilst little difference was observed for larger displacements. As the expected displacements in the wind tunnel were up to $10 \mathrm{~mm}$, this speckle pattern was printed onto decal-paper and attached to the surface of the foil at the tip as shown in Figure 3.

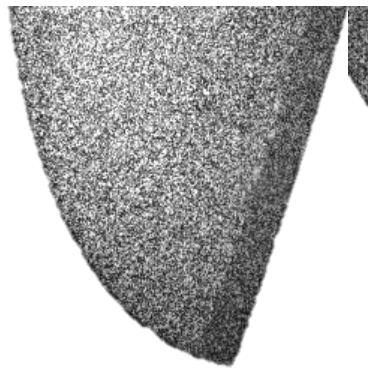

(a)

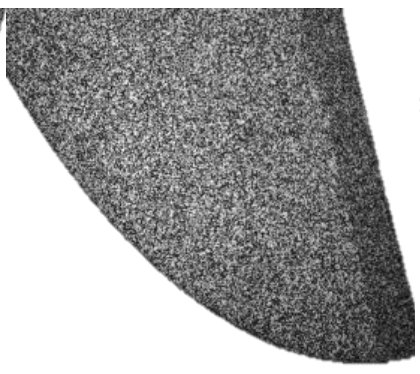

(b)

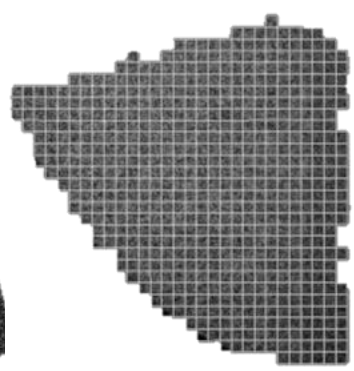

(c)

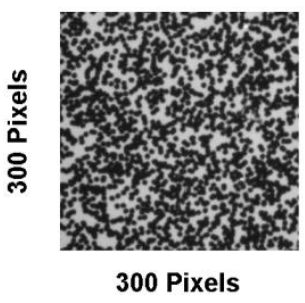

(d)

Figure 3: Speckle pattern on tested foil. Left camera view(a), right camera view(b), mapped image from DaVis providing the calabrated region of the specimin from both camera images(c) and zoomed-in speckle pattern (d). In all images the leading edge of the foil is on the left and the trailing edge is on the right.

Two MANTA G-504B/C cameras were placed behind open fairings on an instrument rail mounted on the floor of the wind tunnel. This removed any possibility of image distortion associated with cameras looking through viewing windows. For the system to operate with large out of plane deflections and dynamic motions, a small aperture and shutter speed were required to increase the depth of field and reduce specimen motion induced blurring for unsteady deflections. Therefore high lighting levels were required to provide maximum sub-pixel contrast of the speckle pattern on the foil. A high-powered LED light source was mounted on the rail between the two cameras. Two additional high powered LED lights were located outside the tunnel and directed through a viewing window, seen in Figure 2. The equipment used in the experiment is described in Table 1. 
Table 1: Digital Image Correlation equipment and settings.

\begin{tabular}{|c|c|}
\hline Equipment & Set-up \\
\hline \multirow{7}{*}{ Camera } & 2 MANTA G-504B/C \\
\hline & Sensor size: $8.5 \times 7.1 \mathrm{~mm}$ \\
\hline & Pixel size: $3.45 \mu \mathrm{m}$ \\
\hline & Resolution (max): 2452 x 2056 pixels \\
\hline & Magnification: 0.15 \\
\hline & Exposure time: $8000 \mu \mathrm{s}$ \\
\hline & Frame rate: $1 \mathrm{~Hz}$ \\
\hline \multirow{2}{*}{ Lens } & Nikon: Nikkor $50 \mathrm{~mm}$ f/1.8D \\
\hline & Aperture: $f-11$ \\
\hline \multirow{2}{*}{ Light } & $1 \times 8$ high power LED light source \\
\hline & 2 x NILA Zaila LED light \\
\hline \multirow{2}{*}{ Speckle pattern } & Speckle size: $0.75 \mathrm{~mm}$ (approx 6 pixels) \\
\hline & Dimensions: 250 x $270 \mathrm{~mm}$ \\
\hline
\end{tabular}

Prior to the experiment, an assessment was made of the available lenses and the resulting field of view within the constraints of the wind tunnel dimensions. To ensure that the specimen remained in focus during the experiments it was important to calculate the expected depth of field for a given lens, calculated as:

$$
D o F=D_{F}-D_{N}=\frac{2 f^{2} s^{2} N c_{b}}{f^{4}-s^{2} N^{2} c_{b}{ }^{2}}=\sim 180 \mathrm{~mm}
$$

where $D_{F}$ is the distance from the camera to the far limit of the depth of field, $D_{N}$ is the distance to the near limit, $s$ is the specimen distance from the camera $(1763.2 \mathrm{~mm}), c_{b}$ is an assumed blur circle of 2 pixels, $f$ is the focal length and $N$ the $f$-number (Jacobson, et al. 2000). As the foil is rotated through a 25 degree range of angles of attack the leading and trailing edges move $52 \mathrm{~mm}$ closer or further away from the cameras. A maximum foil deflection of $15 \mathrm{~mm}$ provides a possible range of out of plane motion of $120 \mathrm{~mm}$, a value within the depth of field range from equation (1).

Figure 4 defines schematically the distances from the cameras and the foil tip on which the speckle pattern is applied. Following the experimental analysis described in Ke, et al. (2011) and the guidance notes in Sutton, et al. (2009), a stereo angle of $\theta=45^{\circ}$ was chosen to maximise the resolution of the out-of plane deflections whilst minimising the in-plane displacement errors and allowing a 25 degree range in angle of attack without moving the cameras. 


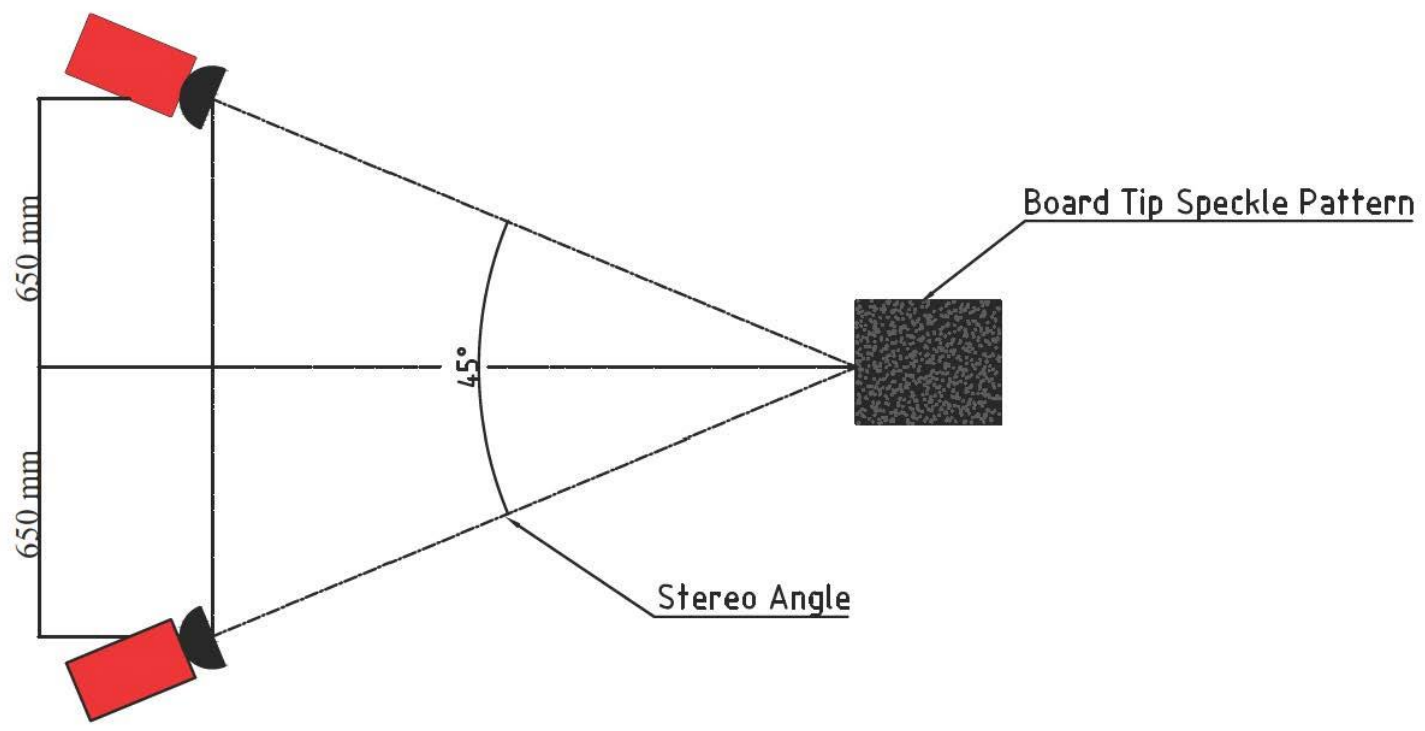

Figure 4: DIC stereo arrangement, providing camera separation distances and angles from the speckle pattern.

The DIC system was calibrated for each angle of attack using a type 11 LaVision calibration plate (Figure 5) positioned in the centre of the speckle pattern. Images from the two MANTA cameras were acquired simultaneously using the imaging toolbox in LabVIEW (IMAQdx). A series of calibration images were taken with the plate at different angles to the foil allowing the LaVision DIC software DaVis 8.1.4 to calculate the position of the two cameras with respect to the specimen. The calibration plate has precisely machined dimensions, with two different levels of markers. The calibration process locates the markers providing the orientation of the plate in each calibration image. The first calibration image is taken with the calibration plate flat on the surface of the specimen and provides the camera coordinate system $\left(x_{c}, y_{c}, z_{c}\right)$ for the DIC deflections. The calibration process provides an RMS fit error for the marker positions on the plate at different orientations. During the first set of experiments, five different images were recorded for each calibration in normal, tilted-down, tilted-up, tilted-right and tilted-left positions. This type of calibration gave, at each angle of attack, an average RMS error value of 0.3 pixels. To investigate the impact of the number of calibration images three more views were captured tilting the plate diagonally (ie both up and to the left and conversely down and to the right) to see if this improved the calibration process. A maximum RMS value of 0.2 pixels was achieved, indicating the calibration accurately determined the camera positions and that including additional calibration images appears to improve the level of accuracy. Figure 5b shows the calibration plate mapped image created within the calibration of the system to check that all the corrected images coincide. A grid of the ideal regular grid of marks is overlaid on the image showing where the centre of all the marks should pass. 

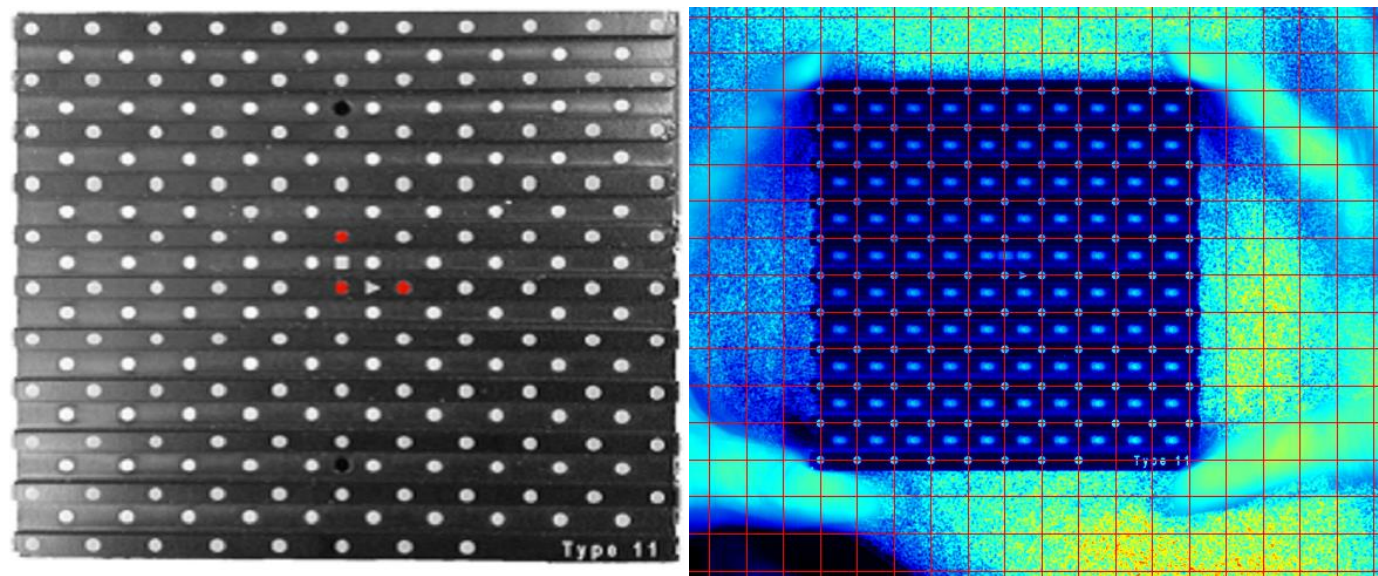

Figure 5: LaVision calibration plate (a) and automatic marker recognition during calibration process within DaVis (b).

Two sets of 30 images were recorded for each wind condition and angle of attack. The tip deflection was calculated by taking the mean deflection within a box located at the foil structure's tip (depicted later in figure 13). Immediately after each calibration a series of 10 no-load images (wind speed: $V_{s}=0 \mathrm{~m} / \mathrm{s}$ ) were acquired to allow the system resolution to be determined. As the foil is not loaded the calculated displacements are due to the system error. The tip deflection error is assessed as the standard deviation of the deflection within the tip box for each image at zero wind speed. The resolution of each component of tip deflection is calculated as the average error over all 10 images. This produced a system resolution for the tip deflection magnitude of $0.023 \mathrm{~mm}$. An additional two sets of images were obtained for each experimental condition on a different day to assess the repeatability of the experimental method.

\section{Results}

\subsection{DIC System Assessment}

An assessment of the accuracy of the DIC system was performed both in a laboratory environment and in the wind tunnel. Before conducting the experiments in the wind tunnel the out of plane accuracy of the 3D DIC system was evaluated in a static laboratory environment. The same speckle pattern used in the wind tunnel was mounted on a flat plate where the out of plane deflection was set using a Vernier scale micrometer with a resolution of $0.05 \mathrm{~mm}$ (Soubeyran 2013). The cameras were set up with approximately the same configuration as in the wind tunnel and a stereo angle of 43.3 degrees. As can be seen in Figure 6 the DIC system calculated the deflection with less than $1 \%$ error over a range of deflections from $0.5-25 \mathrm{~mm}$. However for deflections below $0.5 \mathrm{~mm}$ the error increases significantly. From previous static loading of the tested foil conducted by Soubeyran (2013) the deflections in the wind tunnel were expected to be up to $10 \mathrm{~mm}$ at $40 \mathrm{~ms}^{-1}$, providing low displacement error with this DIC configuration. 


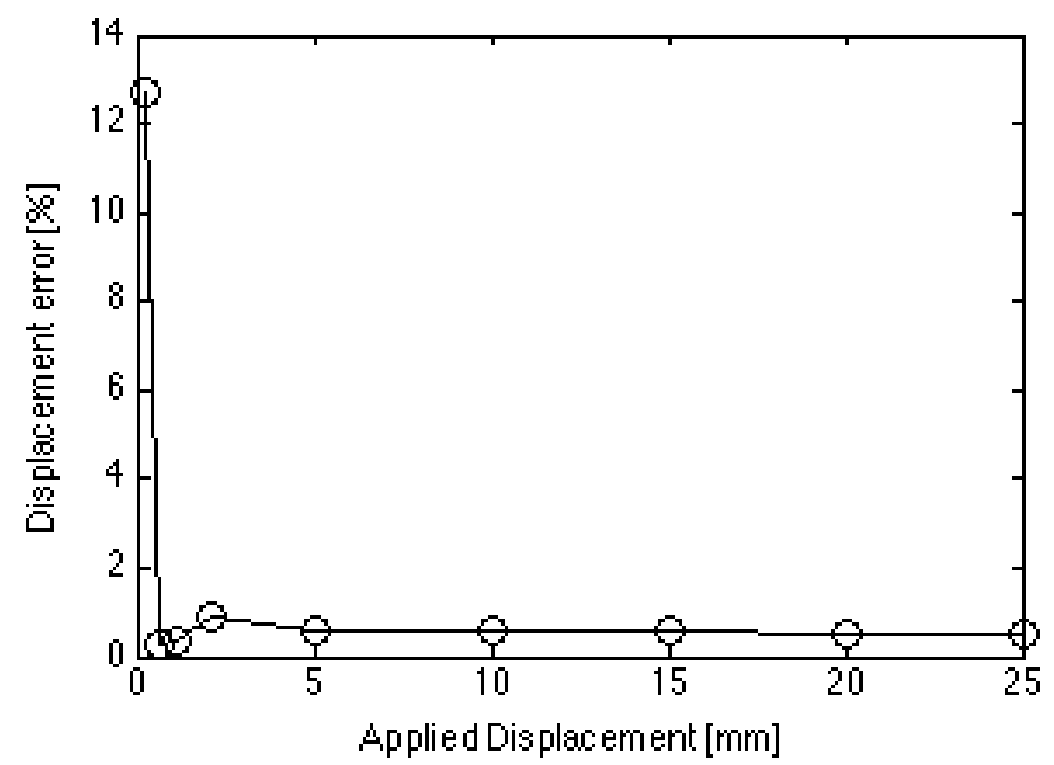

Figure 6: DIC displacement error for a flat plate moved through known displacements within a Lab environment.

The laboratory study gave confidence in the general experimental configuration and speckle pattern validity, however it did not assess the impact of the 3-D surface of the foil at the tip. Moreover, it did not account for the exact distances encountered in the wind tunnel and the angle of the foil with respect to the cameras. Therefore, an aerofoil-shaped structure was placed on a micrometer within the wind tunnel in the same orientation as the tested structure and at exactly the same distances to the cameras. Out-of plane displacement were applied from 0.5 to $19 \mathrm{~mm}$ to assess the validity of the DIC equipment to capture deflections for a 3-D curved surface, Figure 7a.

The DIC displacements are compared with the known displacements to provide a percentage error in Figure 7b. The figure shows the variation in displacement error for a range of different subset sizes used within the DIC processing. For the largest subset size the DIC error is less than $0.8 \%$ for all the displacements assessed confirming that the DIC methodology retains a high degree of accuracy when applied to curved surfaces.
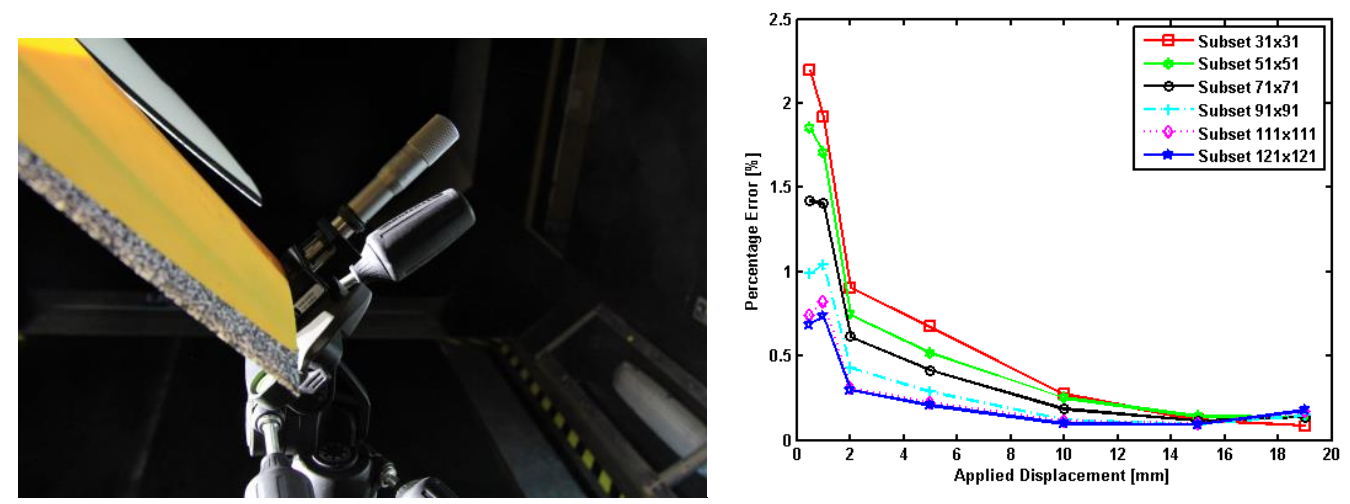

Figure 7: (a) Aerofoil-shaped structure, positioned on a micrometer, on the same plane as the tip of the tested foil (i.e. $37^{\circ}$ from horizontal). (b) Out of plane DIC displacement error, for known displacements of a curved aerofoil specimen in the wind tunnel. 
The subset size determines the spatial resolution on which the displacement vectors are calculated. Increasing the subset size reduces the error in rigid body displacements as a larger number of pixels are included in each interrogation widow providing more data to correlate. However this reduces the spatial resolution, smoothing out high displacement gradients associated with looking at strains in joints or small component failure loadings (Pan, et al. 2008). As large displacement gradients are not expected within the tip region of the foil a maximum subset size of $121 \times 121$ pixels was chosen for the experiments.

\subsection{Wind Tunnel Tests}

During the experiment, fifteen test cases were investigated varying the wind speed and the angles of attack for a combined assessment of DIC and aerodynamic loadings. For each wind speed $\left(V_{s}=20,30\right.$ and $40 \mathrm{~ms}^{-1} ; R e=3.3,5$ and $\left.6.6 \times 10^{5}\right)$, five angles of attack $(\alpha=0,3.5,8.5,13.5$ and 18.5 degrees) were tested. These conditions represented the maximum range of fluid loading achievable for both fully attached and stalled conditions. The images were recorded at an acquisition rate of $1 \mathrm{~Hz}$. The low acquisition rate was related to the capabilities of the DIC system. Initially the DIC system was tested over a wider range of wind speeds $\left(V_{S}=10,20\right.$ and $\left.40 \mathrm{~ms}^{-1}\right)$ to assess the impact of the airflow on the cameras.

\subsubsection{Impact of Aerodynamic Vibration}

Once the DIC methodology had been assessed in a static environment, it was necessary to investigate the effect of aerodynamic loads on the DIC measurements. Preliminary dynamic tests revealed a large amount of image blurring at high wind speeds. The possible causes for this could emanate from camera vibration and/or dynamic vibration of the tested structure due to flow separation and reattachment. The principle source of blurring was shown to be camera vibration by observing the same effect while focusing the two cameras on the rigid wall of the wind tunnel.

The fluid motion around the cameras was assessed using wool tufts on the fairing upstream of the cameras and on the cameras themselves. At the higher wind speeds it was possible to see large turbulence eddies downstream of the open fairing, as can be seen in Figure 8. To eliminate the flow-induced vibrations, enclosures for the cameras were manufactured.

Turbulent motion of tufts

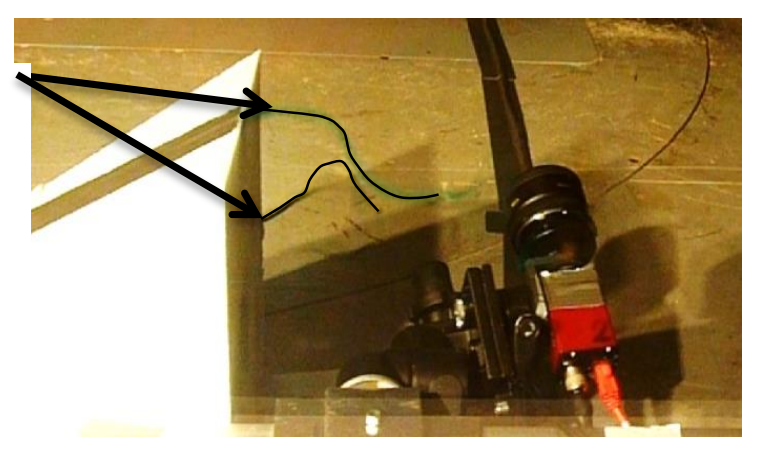

Figure 8: Unsteady fluid regime around a camera behind an open fairing within the wind tunnel. 
The tunnel was then run for the same test with the camera enclosed in the fairings, producing a significant reduction in image blurring. For each wind speed the first image of the set was taken as the reference picture and compared to the next 9 images of that condition. This allowed the vibration displacement to be assessed independently of the mean foil deflection. The $1 \mathrm{~Hz}$ DIC frame rate meant that the vibration displacement could not be fully characterised. However by analysing the standard deviation of the vibration displacement it was possible to quantify the improvement in the mean deflection measurements obtained by enclosing the cameras in closed fairing, see Figure 9.

An average improvement of $70 \%$ in vibration induced displacement was recorded comparing the vibration magnitude in both $y$ and $\mathrm{z}$ direction for the same wind conditions (i.e. $V_{s}=20$ and $40 \mathrm{~ms}^{-1}$ ). However, it is not removed entirely and still increases with wind speed. This could indicate that there is some vibration of the structure at an angle of attack of 5 degrees, despite none being observed by eye. Another potential source of error could come from vibration in the wooden panelled floor of the tunnel. These panels may transfer some vibrations to the aluminium instrument rails to which the cameras were attached. Therefore for the rest of the experiments the cameras were mounted onto rails on the rolling road, isolating them from the main wind tunnel structure.

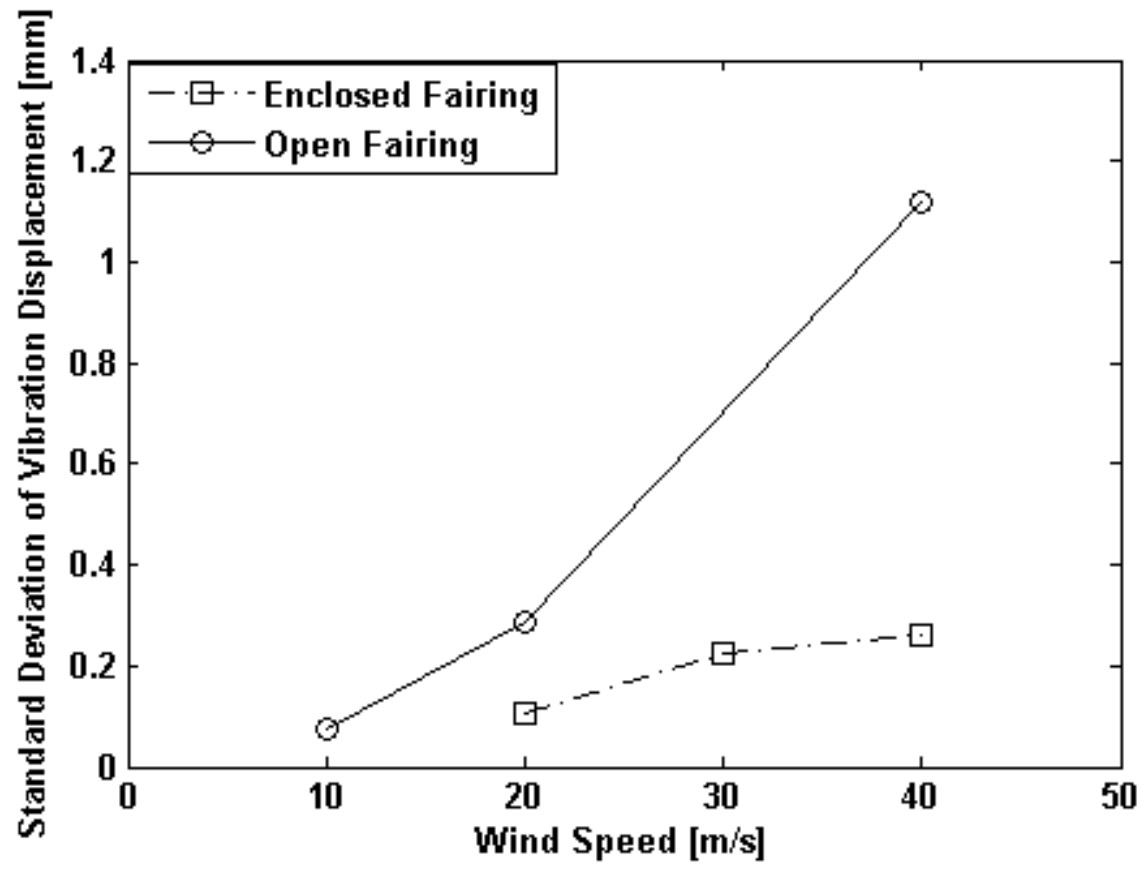

Figure 9: Effect of camera fairings on the camera vibration induced DIC displacement.

\subsubsection{Fluid loads on foil}

The fluid dynamic forces recorded by the overhead balance (dynamometer axis system $\left.x_{d}, y_{d}, z_{d}\right)$ were converted into the tunnel axis system based on the yaw angle of the dynamometer (which is the same as the angle of attack of the foil). The drag, side and vertical force coefficients ( $C_{D}, C_{Y}$ and $C_{Z}$ respectively) can be calculated as;

$$
C_{D}=\frac{F_{y_{d}} \sin (\alpha)+F_{x_{d}} \cos (\alpha)}{0.5 \rho c L V_{s}^{2}}, C_{Y}=\frac{F_{y_{d}} \cos (\alpha)-F_{x_{d}} \sin (\alpha)}{0.5 \rho c L V_{s}^{2}} \text { and } C_{z}=\frac{F_{z_{d}}}{0.5 \rho c L V_{s}^{2}}
$$


where $F_{\left(x_{d}, y_{d}, z_{d}\right)}$ are the dynamometer forces, $\alpha$ is the angle of attack of the foil, $\rho$ is the air density, $c$ is the foil chord, $L$ is the foil span (measured as a straight line from the foil trailing edge as it exits the hull to the foil tip) and $V_{s}$ is the wind speed.

The air temperature and atmospheric pressure were used to calculate the air density to remove the effect of the air being heated up by the work done by the fan. It should be noted that the fluid forces in air are approximately one fifth of those in water at Reynolds number equivalence due to the change in density. A greater number of angles of attack were investigated measuring just aerodynamic forces to check the foil alignment in the tunnel. Zero side force was measured at an angle of attack of 1.5 degrees, indicating a slight misalignment of the foil from the dynamometer. The recorded angles of attack have therefore been corrected to account for this. The resulting force coefficients can be seen in Figure 10, indicating flow separation starting to occur at $\alpha=13.5^{\circ}$ causing the rate of increase in side and vertical force to drop off and the drag to increase.. In a post stall condition, a variation in the force coefficients with wind speed is also observed indicating that the deformation of the structure is affecting the aerodynamic performance of the foil.

The time history of the forces at an angle of attack of 20 degrees reveals a large variation in the vertical force compared to the sideways lift force. A large variation in the DIC deflection results is also observed in these cases, which indicates unsteady foil motions. This is discussed in more detail in section 3.2.3. The greater variation in vertical force is due to the curved nature of the foil providing more vertical force near the tip. Any foil motion is typically greatest at the tip causing larger variations in vertical force. The frequency of the aerodynamic force variation was $18 \mathrm{~Hz}$ which was also measured as the natural frequency of the specimen, determined from the oscillations in force after hitting the clamped foil in still air.

The recorded moments and forces can be used to calculate the centre of effort of the fluid forces on a known plane within the wind tunnel. The curved surface of the foil was simplified to a plane passing through the exposed foil root and tip, parallel with the dynamometer $x$-axis. This procedure provided the attachment point of the static weights within $2 \%$ accuracy and can therefore be used to indicate how the pressure distribution changes over the foil. The variation in centre of effort during the experiments can be seen in Figure 11. It can be seen that the span wise centre of effort remains fairly constant for different angles of attack, whereas significant changes are observed in the chord-wise direction. The most obvious change is the centre of effort moving towards the trailing edge when the foil stalls (above $\alpha=15 \mathrm{deg}$ ). This is due to a significant change in the pressure distribution as the flow separates from the suction side of the foil. We can also see the impact of foil deflection with increased wind speed before stall, while the flow is still attached. It should be noted that the approximation of the pressure distribution to a single location will not capture the full complexity of the changes over the foil however they can provide a useful indication. 
(a)

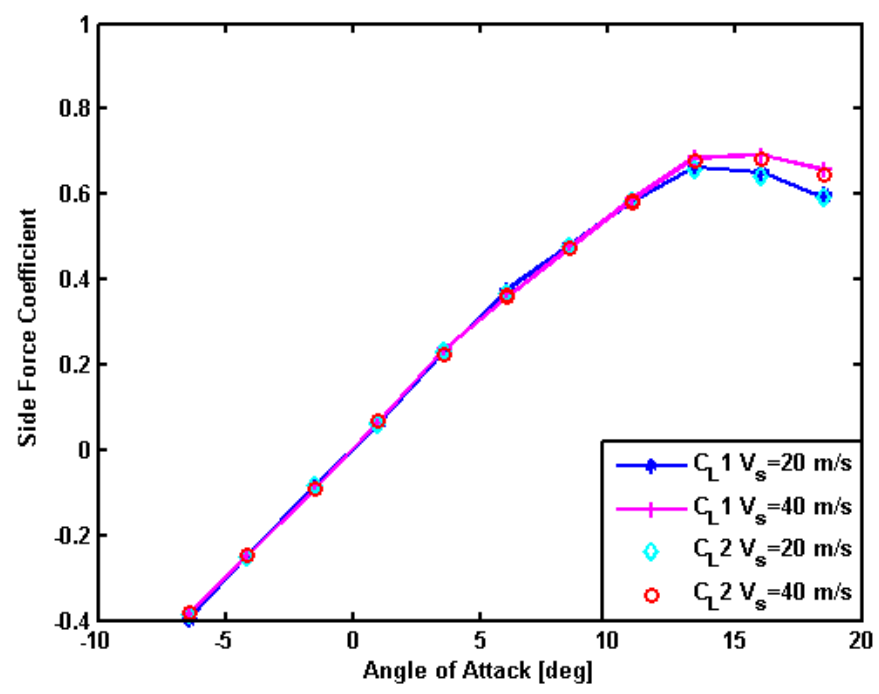

(b)
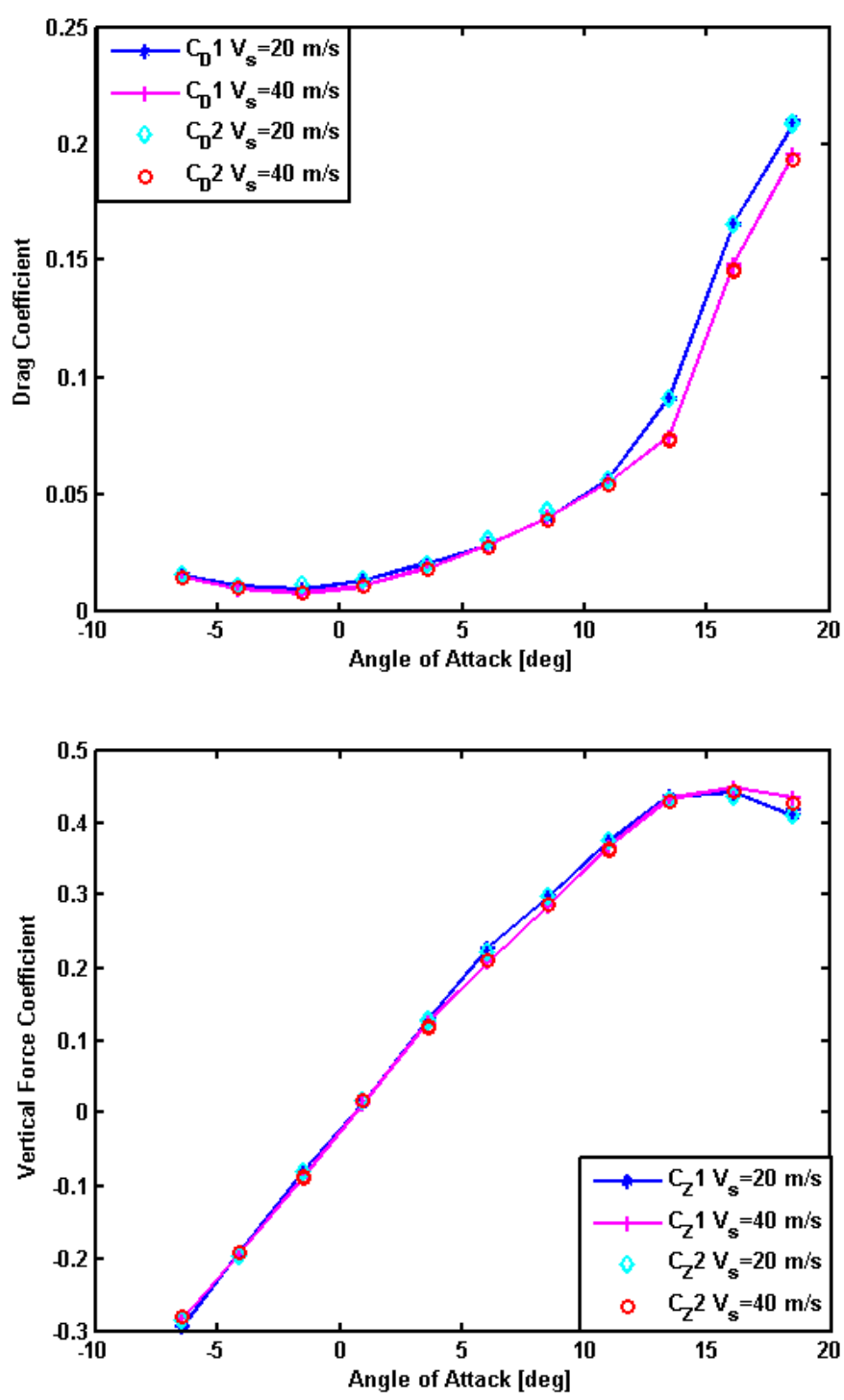

Figure 10: Side, drag and vertical force coefficients of the specimen loaded under two different wind speeds and a range of angles of attack. Repeat values are displayed as additional markers. 

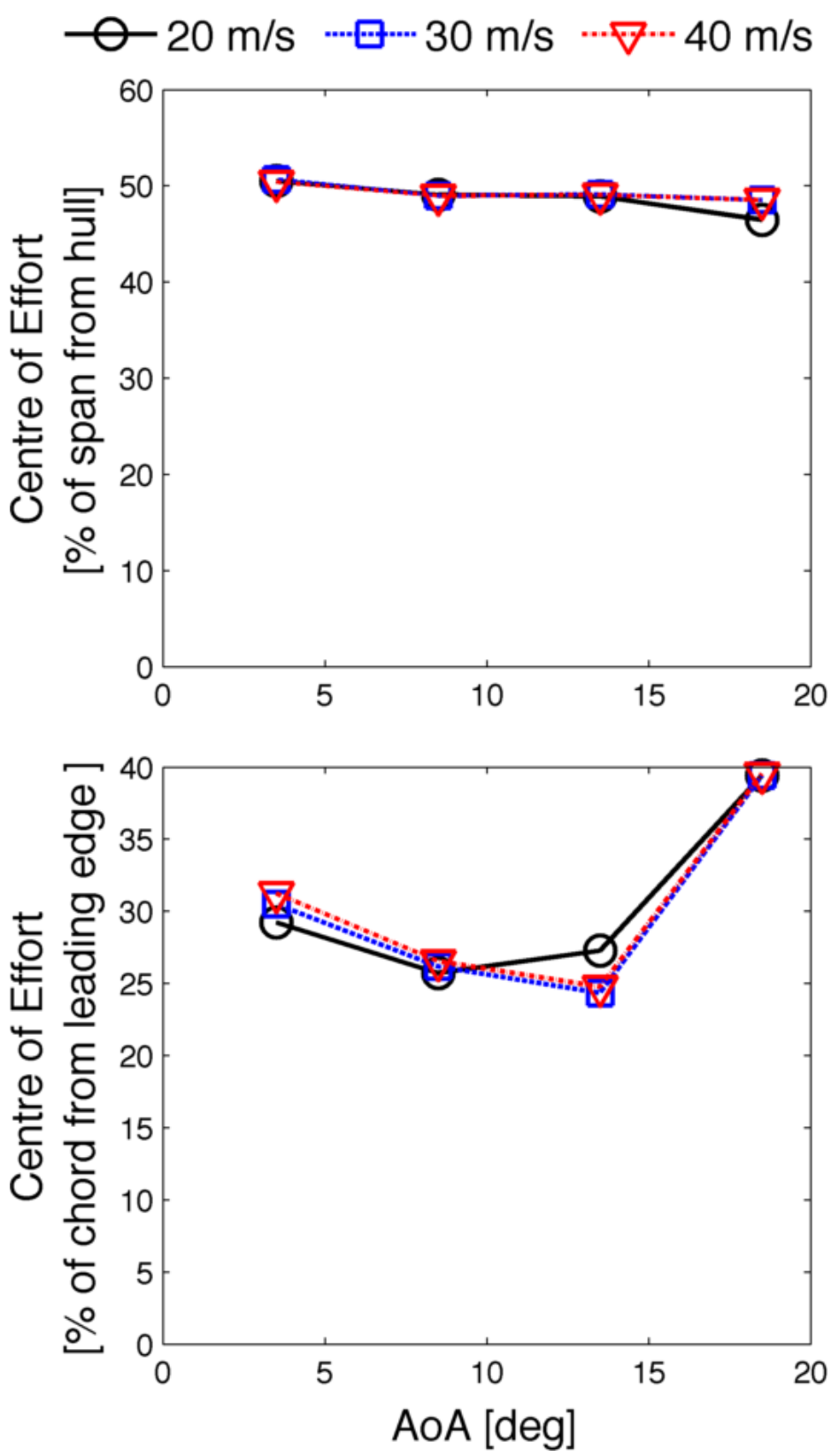

Figure 11: Variation of the calculated centre of effort, of the measured fluid force, with wind speed and angle of attack.

\subsubsection{Structural response to fluid load}

An example of DIC displacement normal to the foil (camera coordinate system, $z_{c}$ ) for different wind speeds is shown in Figure 12. The region in which the tip deflections are inspected is shown as well as the lines at which the twist angle is investigated. The deflected shape represents the foil as mapped by the two cameras. It is possible to note that the displacement increases with the wind speed and along the chord toward the trailing edge. Furthermore, the higher displacement level is observed at the tip of the foil. For a wind speed of $V_{S}=0 \mathrm{~m} / \mathrm{s}$ the displacement is almost zero and the displayed values represent the noise in the system. The apparent differences in deflection between the centre and the edges of the unloaded specimen only represents the resolution of the system 
and the calibration methodology, a higher accuracy may be achieved with a calibration plate that covers the entire region of interest.
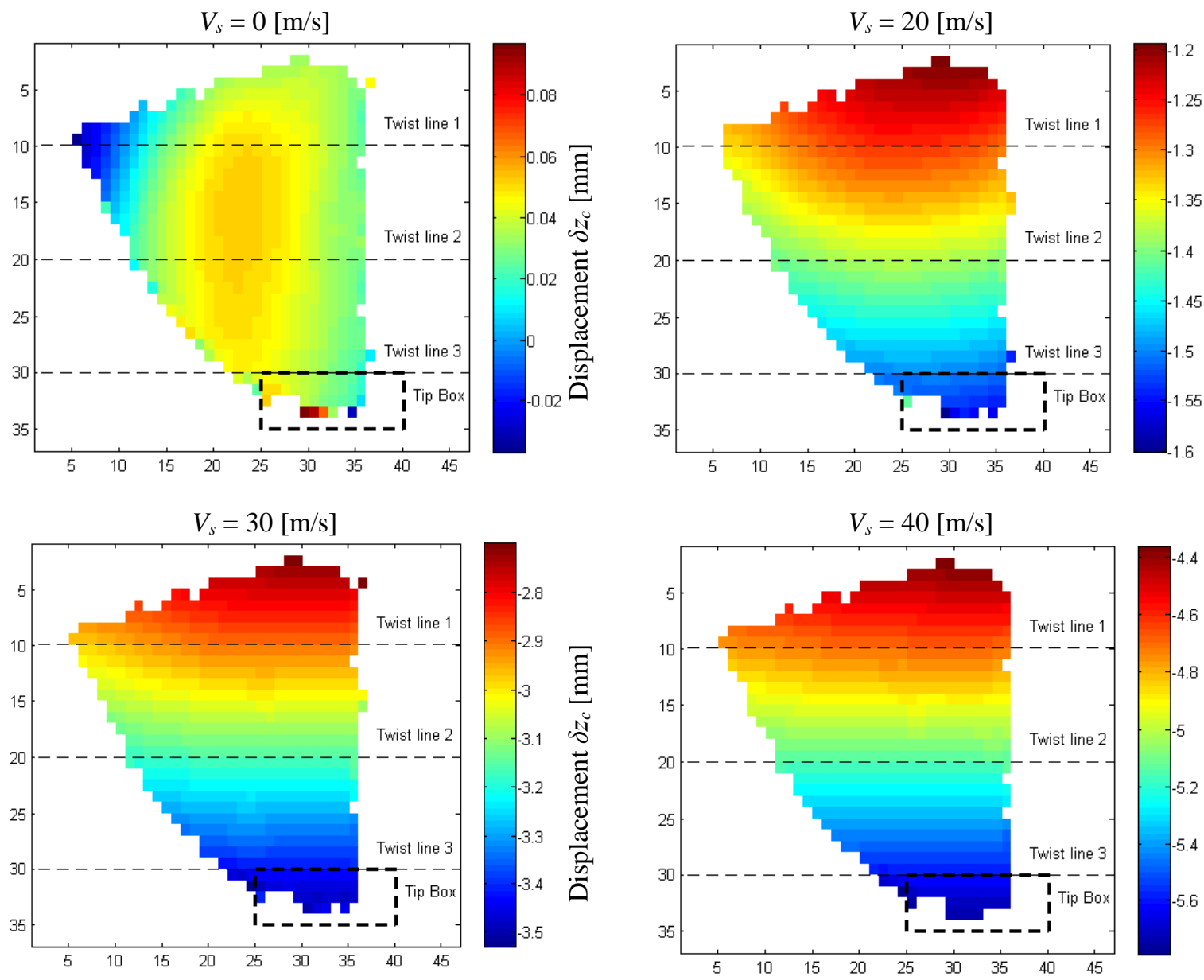

Figure 12: Example of the full field, out of plane, displacement (camera coordinate system $\left.\delta z_{c}\right)$ for a single angle of attack at different wind speeds $\left(V_{s}\right)$. The foil leading edge is located on the left of each image. Note displacement scale changes for clarity. Defined locations for obtaining twist and tip deflections are also displayed.

The tip deflection magnitude is calculated from the tip deflections $\delta y_{c}$ and $\delta z_{c}$ obtained from the DIC analysis. Figure 13 shows the deflection response of the structure for different loading conditions including fluid forces and static calibration weights applied to the foil. In general the deflections show a linear increase in deflection with respect to the normal force applied. The difference in slope between the static load (labelled as calibration weights) and dynamic data is due to the differences in load condition from a single point load to a dynamic pressure distribution. The weights in the static condition were applied with a pulley system at the estimated centre of effort (25\% of the chord from the leading edge of the foil and $43 \%$ of the span from the bottom of the hull). These differences highlight the need to apply real fluid loadings during FSI investigations. As described in section 3.2.2, the fluid forces in air are significantly lower than the experienced forces in water; therefore the measured deflections are smaller than those 
expected in the corresponding sailing condition. These small deflections make this a challenging assessment test case for the DIC methodology.

In order to assess the instantaneous variability of the system the mean deflection obtained from the two sets of 30 images, acquired sequentially for each condition, were compared within both Figure 13 (a) or (b). Close agreement between data sets within each figure provides confidence in the values obtained. The repeatability of the experimental set up was assessed by repeating all the investigated conditions on a different day. The comparison of this data can be seen by comparing Figure 13 (a) and (b). Despite small variations between the data sets very good agreement is seen when repeating conditions on a different day.

(a)

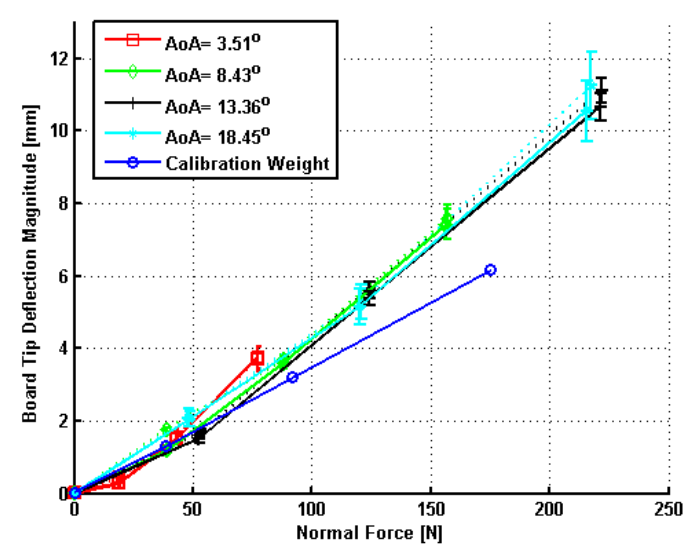

(b)

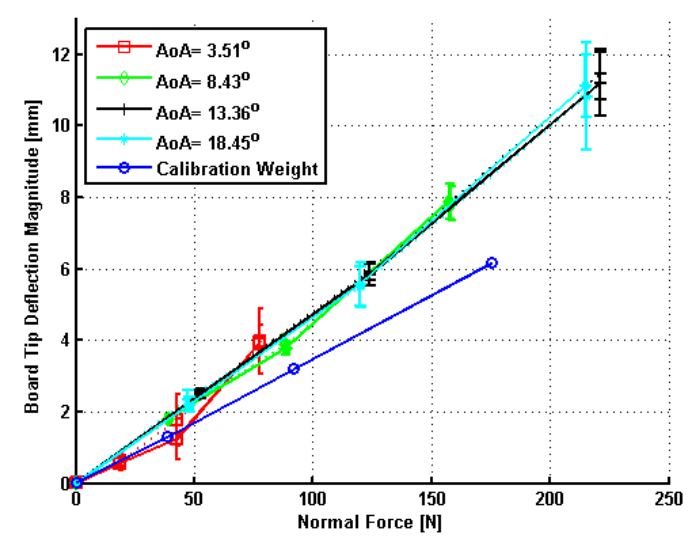

Figure 13: Tip displacement magnitude calculated with DIC at different aerodynamic force magnitudes for the full range of angles of attack and wind speeds conducted on Day 1 (a) and repeated on Day 2 (b). In both figures the mean deflection from the first set of images is displayed as a solid line with the second set as a dashed line. The error bars represent the standard deviation of the 30 images in each set.

The accuracy and repeatability of the DIC system can be observed in the consistent repeat values and generally low standard deviation for angles of attack below stall. For higher angles of attack there is a noticeable increase in the standard deviation. Table 2 presents the results for each condition tested. The standard deviation represents both the system repeatability and an indication of the dynamic motions of the specimen.. In order to characterise the unsteady structural response of the specimen within the time domain, there is a need for a higher number of images per second in the DIC methodology.

Figure 14 presents the standard deviation of the foil tip deflection. As previously discussed, the condition for an angle of attack of $\alpha=3.5^{\circ}$ did not appear to have any foil vibration, therefore the standard deviation provides a measure of the system error, potentially due to camera vibrations. However, as the cameras motions will be the same for any given wind speed any increase in standard deviation due to changing the angle of attack must be due to dynamic foil motions. The large increase in standard deviation observed for an angle of attack of $\alpha=18.5^{\circ}$ indicates an unsteady structural response associated with fluctuating fluid forces due to flow separation during stall. The standard deviation of 30 samples cannot determine the maximum magnitude or frequency of the 
board motion however it does provide a useful indication of when dynamic motions are occurring and a measure of their relative magnitude. This allows useful comparisons to be made with the flow regime and highlights the limitation of the current DIC methodology as unsteady structural responses can only be identified but not analysed.

Table 2: Tip deflection data

\begin{tabular}{|c|c|c|c|c|c|}
\hline $\begin{array}{c}\text { AoA } \\
\text { [degrees] }\end{array}$ & $\begin{array}{c}\text { Wind } \\
\text { Speed } \\
{[\mathrm{m} / \mathrm{s}]} \\
\end{array}$ & $\begin{array}{c}\text { Normal } \\
\text { Force } \\
{[N]} \\
\end{array}$ & $\begin{array}{c}\text { Tip Deflection } \\
\text { Magnitude } \\
{[\mathrm{mm}]} \\
\end{array}$ & $\begin{array}{c}\text { Mean Standard } \\
\text { Deviation } \\
{[\mathrm{mm}]} \\
\end{array}$ & $\begin{array}{c}\text { Coefficient of } \\
\text { Variation of } \\
\text { Deflection } \\
\end{array}$ \\
\hline \multirow{4}{*}{0.09} & 0 & 0.09 & 0.00 & 0.01 & \\
\hline & 20 & 2.17 & 0.06 & 0.19 & 2.95 \\
\hline & 30 & 4.63 & 0.46 & 0.47 & 1.01 \\
\hline & 40 & 7.54 & 0.98 & 0.58 & 0.59 \\
\hline \multirow{4}{*}{3.6} & 0 & 0.16 & 0.01 & 0.03 & \\
\hline & 20 & 18.71 & 0.58 & 0.19 & 0.33 \\
\hline & 30 & 42.78 & 1.51 & 0.64 & 0.43 \\
\hline & 40 & 77.74 & 3.92 & 0.73 & 0.19 \\
\hline \multirow{4}{*}{8.52} & 0 & 0.25 & 0.00 & 0.01 & \\
\hline & 20 & 39.06 & 1.80 & 0.09 & 0.05 \\
\hline & 30 & 88.61 & 3.84 & 0.24 & 0.06 \\
\hline & 40 & 157.97 & 7.86 & 0.49 & 0.06 \\
\hline \multirow{4}{*}{13.45} & 0 & 0.26 & 0.01 & 0.01 & \\
\hline & 20 & 52.55 & 2.50 & 0.11 & 0.04 \\
\hline & 30 & 123.80 & 5.88 & 0.27 & 0.05 \\
\hline & 40 & 221.13 & 11.32 & 0.80 & 0.07 \\
\hline \multirow{4}{*}{18.54} & 0 & 0.30 & 0.00 & 0.00 & \\
\hline & 20 & 47.62 & 2.26 & 0.24 & 0.11 \\
\hline & 30 & 119.94 & 5.54 & 0.58 & 0.11 \\
\hline & 40 & 215.10 & 10.97 & 1.20 & 0.11 \\
\hline
\end{tabular}

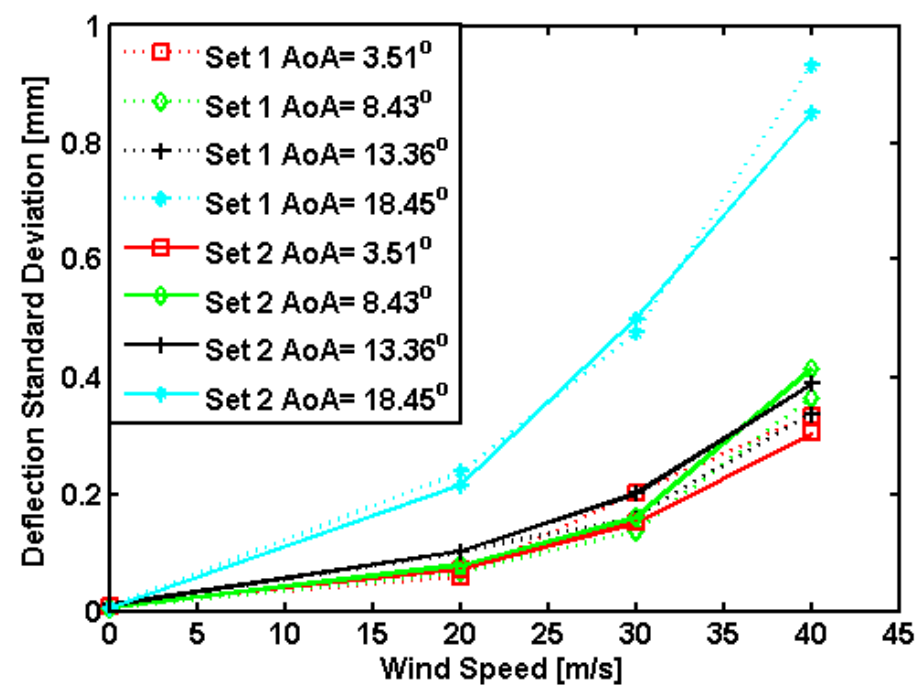


Figure 14: Variability in DIC deflection data displayed as standard deviation of foil tip deflection for different angles of attack and wind speed. Repeat values are presented with different line styles.

\subsubsection{Blade twist angle variation}

The impact of the deflection on the blade twist was assessed by measuring the out of plane deflection along lines running perpendicular to the trailing edge, as shown in Figure 12. At span locations $80 \%, 88 \%$ and $97 \%$ from the hull the chord length was 180,150 and $80 \mathrm{~mm}$ respectively as the foil tapers. The local chord length and the difference in normal deflection $\left(\delta z_{c}\right)$ at the leading and trailing edges were used to calculate the local change in angle of attack $(\alpha)$, displayed in Figure 15. As the aerodynamic forces are significantly smaller than the hydrodynamic load the foil is designed for, the observed twist deflections are very small. However, as the aerodynamic load increases with wind speed larger twist deflections are observed as a change in local angle of attack. The magnitude of the twist deflections also tends to increase towards the blade tip ( $97 \%$ of span).

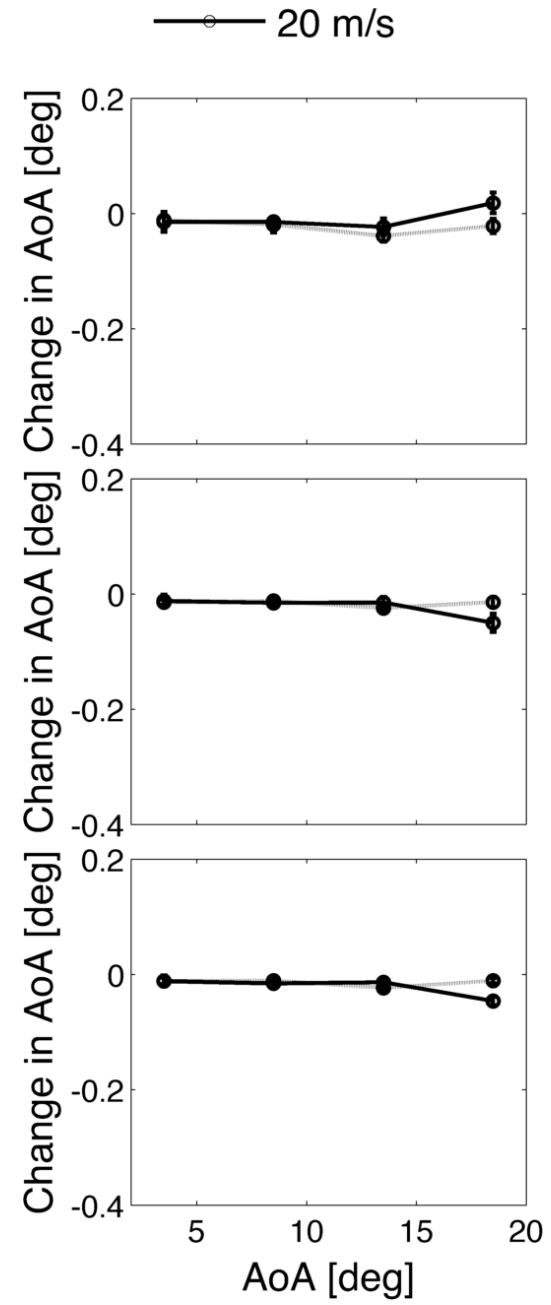

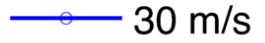
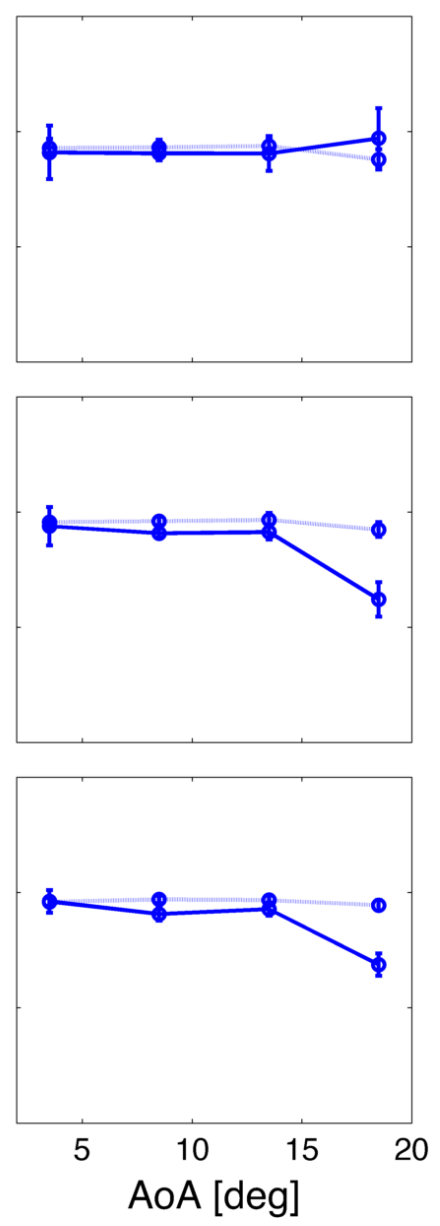

$-40 \mathrm{~m} / \mathrm{s}$
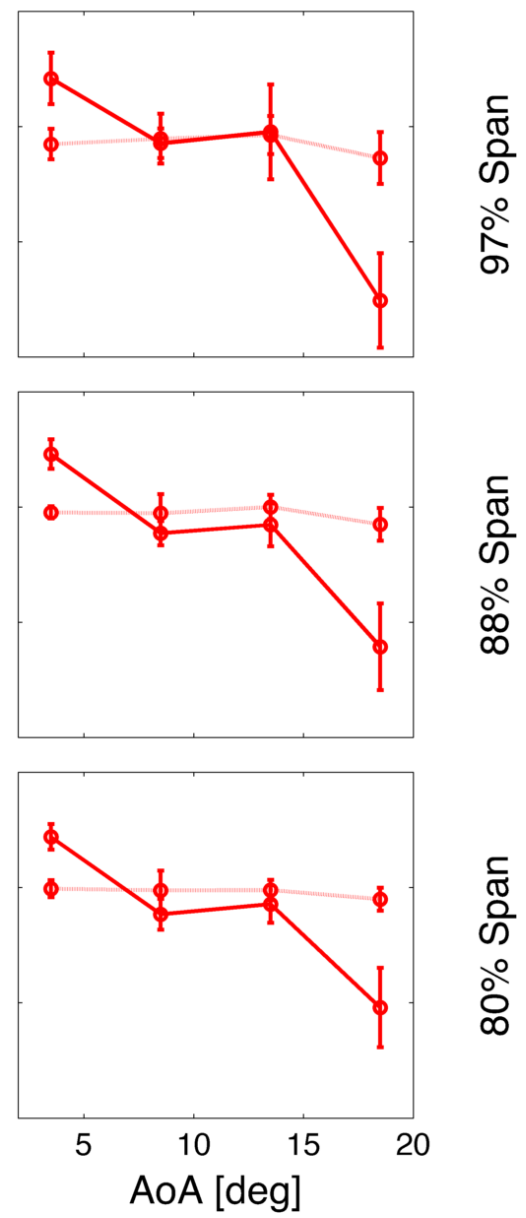

Figure 15: Change in local foil tip angle of attack due to specimen twist for different span locations and initial angles of attack. The repeat data sets from different days are shown by different line styles whilst the standard deviation of each data set is presented as error bars.

Based on an out of plane measurement uncertainty of $0.7 \%$ (see Figure 6) the twist uncertainty due to the measurement accuracy of the DIC system can be estimated using 
the local chord of the blade. For the twist measurements at $88 \%$ of the span the twist uncertainty varies from less than $0.02 \mathrm{deg}$ for $4 \mathrm{~mm}$ of tip deflection (representing attached flow for $30 \mathrm{~m} / \mathrm{s}$ and less) increasing to a maximum of $0.05 \mathrm{deg}$ for $11 \mathrm{~mm}$ tip deflection (representing stalled conditions at $40 \mathrm{~m} / \mathrm{s}$ ).

In general, as the angle of attack, set at the blade root increases, the twist deflection reduces the local angle of attack towards the blade tip. This is most noticeable for the largest angle of attack and corresponds to a significant shift in the centre of effort towards the trailing edge of the foil, observed in Figure 11. This indicates that when the foil stalls the centre of effort moves behind the shear centre of the foil deflecting the trailing edge away from the wind, reducing the local angle of attack of the foil section. It can therefore be assumed that the shear centre is less than $40 \%$ of the chord from the leading edge, providing another potential reason why very small twist angles were observed below stall angles. The decrease in effective angle of attack occurring in the stall condition can be linked to the increase in side and vertical force coefficients with high wind speeds (Figure 10), demonstrating the possible advantages of designing structures to control their behaviour in increased aerodynamic load.

The standard deviation of the twist deflection data is seen to increase with angle of attack, in a similar manner to the tip deflection data. This again indicates structural dynamic motions in the stalled condition, which cannot be characterised with the low DIC acquisition rate. The standard deviation also increases with wind speed, this could be due to camera vibration. However, at $40 \mathrm{~m} / \mathrm{s}$ the standard deviation increases towards the tip suggesting that this variability in the data is due to the structural response, rather than camera motions. As this increase is observed at all angles of attack, the unsteady response may be a function of flow velocity rather than stall. For angles of attack below stall and for lower wind speeds the low standard deviation provides a higher degree of confidence in the twist values obtained, however due to the reduced aerodynamic loading in these cases the twist deflections are very small, potentially lying within the measurement uncertainty of the DIC system.

The repeatability of the experimental procedure can be seen in the two data sets presented from different days. For angles of attack below stall good repeatability is observed in most of the conditions. A much greater variation between repeat values is observed for the stall condition $\left(\alpha=18.5^{\circ}\right)$ that is likely to be caused by the unsteady nature of the structural response in these conditions. This again highlights the limits of the current system regarding unsteady deformations and that a higher camera acquisition rate is required.

The DIC system shows that very little twist deflection is observed below stall. Larger twist deflections are observed at higher angles of attack, however the unsteady nature of these deformations results in greater variability in the measurements and a lack of repeatability between tests. The trends observed in the twist data appear consistent with changes in the measured forces and their centre of effort, however it is noted that the magnitude of some of these changes are similar to the measurement uncertainty for the DIC system. Therefore this technique should be applied to specimens with larger twist 
deformations in the future and higher sampling frequencies should be used for unsteady deformations.

\section{Conclusions}

A methodology involving 3-D DIC has been developed and its performance assessed for the measurement of out of plane deflections of large specimens within a laboratory and a wind tunnel environment (i.e. under static and dynamic loadings). Enclosed camera fairings were shown to reduce the DIC system error by $57 \%$, minimising camera vibration induced deformations. An assessment of different speckle patterns (size and density of the speckles), subjected to known out of plane deflections, provided a selection process that maintained a static displacement error of less than $1 \%$ over the range of expected deformations. Appropriate optics were also selected for the camera stand-off distance to provide a depth of field greater than the expected deformations and the rotation of the foil at different angles of attack. The resulting DIC methodology was shown to have a displacement error of less than $0.8 \%$ for a curved aerofoil specimen for known displacements up to $20 \mathrm{~mm}$.

For the structural arrangement of the specimen the tip displacement was found to vary linearly with fluid load. Small changes in deflection were repeatedly captured by the DIC methodology for steady load conditions before stall was reached. The robustness of the methodology is demonstrated through multiple repeat tests on different occasions. High angles of attack and wind speeds were shown to increase the variation in tip displacement beyond that measured for possible camera vibration. It is concluded therefore that higher sampling rates are required for the DIC system to assess the structural response to unsteady loadings due to a stalled condition.

The full-field nature of the DIC technique has allowed the assessment of the deformation induced blade twist over a large region of a structure at three different span locations near the tip. Crucially full field analysis allows specific areas of interest to be identified from the results and investigated in increased detail, providing greater understanding from a single experimental test. Full field techniques show an advantage over point measurement methods as they are able to provide a methodology that can capture the variation in deflection and twist for small changes over a large distance. The DIC system was shown to repeatedly measure very small deflections for steady flow conditions at angles of attack below stall, however these magnitudes are similar to the measurement uncertainty of the system. As the angle of attack increased the twist deflection tends to reduce the local angle of attack at the blade tip, affecting the aerodynamic performance. This trend is consistent with the measured forces and their centre of effort. However, the unsteady structural response greatly reduces the repeatability in these conditions, requiring higher frame rates to be used in the future.

The current methodology has been shown to provide accurate and repeatable deflection data for aerofoil structures under steady fluid loading. By increasing the DIC acquisition rate this capability could be extended to include assessment of dynamic structural response. 
In future works the developed experimental methodology will be applied to a fully defined structure to generate FSI validation data and a number of tests will be developed incorporating passive adaptive structures in order to investigate an optimised composite lay-up for flexible aerofoil structures.

\section{Acknowledgements}

The authors would like to acknowledge the EPSRC for funding this research under grant number EP/009876/1. The authors would also thank Dave Marshal and his team in RJ Mitchell wind tunnel, the members of the TSRL, LaVision for assistance with DIC methodology and NACRA UK for supplying the tested foil.

\section{References}

Albertani, R., B. Stanford, J.P. Hubner, and P.G. Ifju. 2007. “Aerodynamic Coefficients and Deformation Measurements on Flexible Micro Air Vehicle Wings." Experimentl Mechanics 47: 625-635.

Carpernter, T., C. Ray, and R. Albertani. 2012. "Correlation of Structural Strain to Tip Vorticity and Lift for a MAV Pliant Membrane Wing." In Experimental and Applied Mechanics, Volume 4: Proceedings of the 2012 Annual Conference on Experimental and Applied Mechanics, 1-14. Springer.

Crammond, G., S.W. Boyd, and J.M. Dulieu-Barton. 2013. "Speckle pattern quality assessment for digital image correlation." Optics and Lasers in Engineering 51 (12): 1368-1378.

Fedorov, V. 2012. Bend-Twist Coupling Effects in Wind Turbine Blades. Technical University of Denmark.

Fedorov, V.A., N. Dimitrov, C. Berggreen, S. Krenk, K. Branner, and P. Berring. 2009. "Investigation of Structural Behavior due to Bend-Twist Couplings in Wind Turbine Blades." ICCM.

Ganguli, R., and I. Chopre. November-December, 1995. "Aeroelastic Optimisation of a Helicopter Rotor with Composite Coupling." Journal of Aircraft 32 (6): 13261334.

Helm, J.D., S.R. McNeill, and M.A. Sutton. 1996. "Improved Three-Dimensional Image Correlation for Surface Displacement Measurement." Optical Engineering 35 (0091-3286/96/6.00): 1911-1920.

Jacobson, R.E., S. Ray, Attridge G.G., and N. Axford. 2000. The Manual of Photography. Focal press.

Karaolis, N.M., P.J. Musgrove, and G. Jenimidis. 1988. "Active and Passive Aerodynamic Power Control Using Aswymmetric Fibre Reinforced Laminates for Wind Turbine Blades." Wind Energy Conversation: Proceedings of the 1988 10th BWEA Wind Energy Conference. London.

Ke, X.-D., Schreier H.W., M.A. Sutton, and Y.Q. Wang. 2011. "Error Assessment in Stereo -Based Deformation Measurements." Experimental Mechanics 51: 423441.

Khan, A.M., D.O. Adams, V. Dayal, and J.M. Vogel. 2000. "Effects of Bend-Twist 
Coupling on Composite Propeller Performance." Mechanics of Composite Materials and Structures 383-401.

LaCroix, B.W., J. Gardiner, and P.G. Ifju. 2013. "Investigating the Aerodynamic Effects of Flexible Membrane Wings." In Imaging Methods for Novel Materials and Challenging Applications, Volume 3: Conference Proceedings of the Society for Experimental Mechanics Seres 2013, 345-352. Springer.

Lee, Y.-A., and C.-C. Lin. 2004. "Optimized Design of Composite Propeller." Mechanics of Advanced Materials and Structures 17-30.

Lee, Y.-J., and C.-C. Lin. 2004. "Optimised Design of Composite Propellers." Mechanics of Advanced Materials and Structures 11: 17-30.

Lee, Y-J, Y-T Jhan, and C-H Chung. 2012. "Fluid-Structure Interaction of FRP Wind Turbine Blades Under Aerodynamic Effect." Composites.

Lin, H-J., and W-M. Lai. 2010. "A Study of Elastic Coupling to the Wind Turbine Blade by a Compined Analytical and Finite Element Beam Model." Journal of Composite Materials 44 (23): 2643-2665.

Liu, Z, and Y.L. Young. 2009. "Utilization of bend-twist coupling for performance enhancement of composite marine propellers." Journal of Fluids and Structures 1102-1116.

Maheri, A., S. Noroozi, and J. Vinney. 2006. "Combined Analytical/FEA- Based Coupled Aero Structure Simulation of a Wind Turbine With Bend-Twist Adaptive Blades." Renewable Energy 32 (2007): 916-930.

McCormick, N., and J. Lord. 2012. "Digital Image Correlation For Structural Measurements." Civil Engineering 165 (CE4): 185-190.

Molland, A.F., and S.R. Turnock. 2007. Marine Rudders and Control Surfaces: Principles, Data, Design and Applications. Oxford: Butterworth-Heinemann.

Murugan, S., R. Ganguli, and D. Harursampath. January-February, 2008. "Aeroelastic Response pf Composite Helicopter Rotor with Random Material Properties." Journal of Aircraft 45 (1): 306-322.

Nicholls-Lee, R.F., S.W. Boyd, and S.R. Turnock. 2009. "Development of High Performance Composite Bend-Twist Coupled Blades for a Horizontal Axis Tidal Turbine." 17th International Conference on COmposite Materials. Edimburgh.

Pan, B., H. Xie, Z. Wand, K. Qian, and Z. Wang. 2008. "Study on Subset Size Selection in Digital Image Correlation for Speckle Patterns." Optics Express 16 (10).

Rastogi, P., and E. Hack. 2012. Optical Methods for Solid Mechanics: A Full-Field Approach. Wiley-VCH Verlag GmbH \& Co.

Reu, P. 2012. "Hidden Components of DIC: Calibration and Shape Function - Part1." Experimental Techniques 36: 3-5.

Reu, P. 2013. "Stereo-Rig Design: Stereo-Angle Selection - Part 4.” Experimental Techniques 37: 1-2.

Shirk, M., and T. Hertz. 1986. "Aeroelastic Tailoring- Theory, Practice and Promise." journal of Aircraft 23 (1).

Soubeyran, X. 2013. Deformation Measurement of Multihull Daggerboard Under Realistic Sailing Loads. MSc Thesis, Southampton: University of Southampton. 
Sutton, M.A., J.J. Orteu, and H. Schreier. 2009. Image Correlation for Shape Motion and Deformation Measurements: Basic Concepts, theory and applications. New York: Springer.

Thuwis, G.A.A., R. De Breuker, M.M. Abdalla, and Z. Gurdal. 2010. "Aeroelastic Tailoring Using Lamination Parameters.” Structural and Multidisciplinary Optimization 41 (DOI 10.1007/s00158-009-0437-6): 637-646.

Turnock, S.R., and A.M. Wright. 2000. "Directly Coupled Fluid Structural Model of a Ship Rudder Behind a Propeller.” Marine Structures 13 (1): 53-72.

Veers, P., and G. Bir. 1998. "Aeroelastic Tailoring in Wind-Turbine Blade Applications." Wind Power '98, Americal Wind Energy Association Meeting and Exhibition.

Young, Y.L. 2008. "Fluid-structure interaction analysis of flexible composite marine propellers." Journal of Fluids and Structures 24 (6): 799-818.

Zhengzong, T., L. Jin, X. Zhenzhong, and G. Cheng. 2011. "Large Deformation Measurement Scheme for 3D Digital Image Correlation Method." Optics and Lasers in Engineering 50: 122-130. 